\section{Persistent expression of CXCR5 on plasmablasts in IgG4-related disease}

We read with interest the papers by Fox and Fox, ${ }^{1}$ and Wallace et $a l^{2}$ on IgG4 levels and plasmablasts as a marker for IgG4-related disease (IgG4-RD). We strongly approve their results and have reported plasmablasts lacking RP105 (CD180) in IgG4-RD in your journal. ${ }^{3}$ Recently, we have found an important and additional result about plasmablasts in IgG4-RD.

RP105-negative B cells are assigned as five subsets of activated late B cells (subset 1), early or pre-plasmablasts (subset 2), plasmablasts (subset 3), early plasma cells (subset 4) and plasma cells (subset 5). ${ }^{4}$ However, precise phenotype of RP105-negative $\mathrm{B}$ cells in IgG4-RD was hitherto unknown. ${ }^{5}$ To clarify the phenotype of RP105-negative B cells, multicolour analysis (more than 200 antigens on B cells) has been performed using a flow cytometry in IgG4-RD $(n=10)$, patients with systemic lupus erythematosus (SLE) $(n=11)$ and normal subjects $(n=5)$.

Increased RP105-negative plasmablasts are found in patients with both IgG4-RD and SLE. Interestingly, while early plasma cells (subset 4) and plasma cells (subset 5) are frequently found in the peripheral blood from patients with SLE; these cells are very rare in IgG4-RD. ${ }^{3}$ In IgG4-RD, activated B cells (subset 1), pre-plasmablasts or early plasmablasts (subset 2) and plasmablasts (subset 3 ) from patients with active disease are increased compared with that from patients with inactive disease. ${ }^{3}$ However, the mechanism of differential distribution of these cells in IgG4-RD and SLE has not been clarified.

CXCR5 (CD185) on mature B cells is the main chemokine receptor responsible for the migration of $\mathrm{B}$ cells into secondary lymphoid organs. ${ }^{6}$ In patients with SLE, CXCR5 (CD185) is expressed on subsets $0-2$, but it disappears in subsets 3-5 (figure 1). The border of CXCR5 expression in SLE is at the earlier stages of subset 1 or 2 . However, in patients with IgG4-RD, CXCR5 is still expressed on subset 3 , the population of later stage. CXCR5 expression on RP105-negative B cells in a patient with IgG4-RD was significantly stronger than in a patient with SLE in subsets 0 and 3 (figure 2A). Importantly, the mean fluorescent intensity of CXCR5 on subset 3 , plasmablasts from patients with IgG4-RD with multiple organ involvement were also higher than from normal subjects (figure 2B).
Figure 1 Expression of CXCR5 in RP105-negative B cell subsets from patients with IgG4-related disease (IgG4-RD), patients with systemic lupus erythematosus (SLE) and normal subjects. The border of CXCR5 expression is indicated in red.
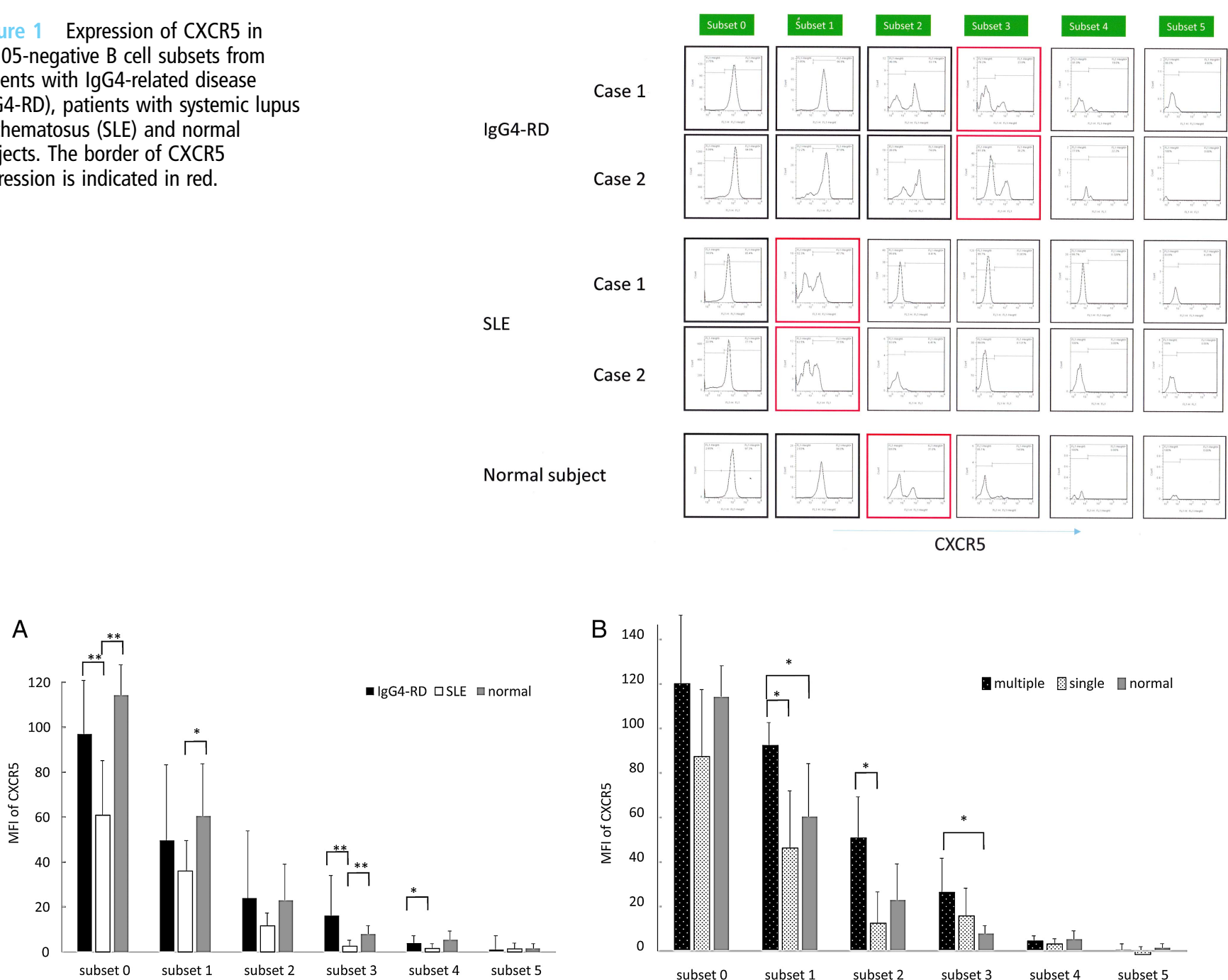

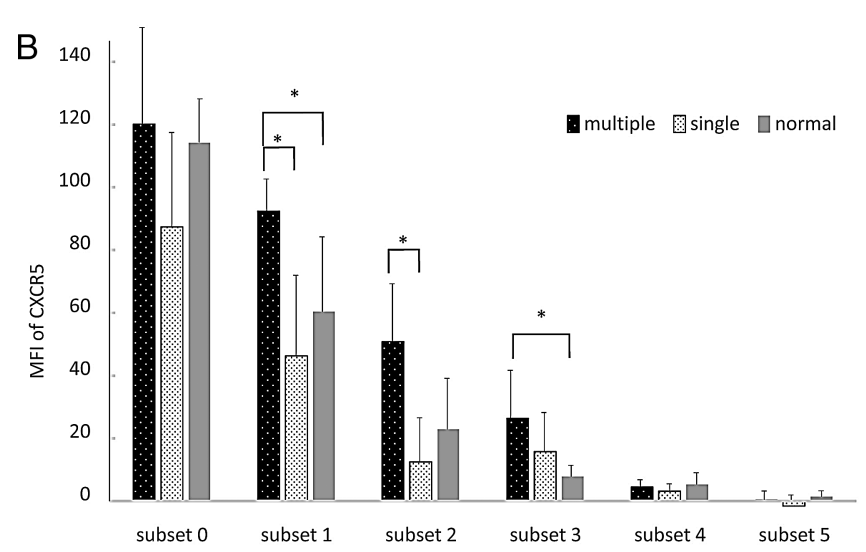

Figure 2 (A) The mean fluorescent intensity (MFI) of CXCR5 on RP105-negative B cells subsets in different patients groups: patients with IgG4-related disease (IgG4-RD), patients with systemic lupus erythematosus (SLE) and normal subjects. ( $\left.{ }^{*} p<0.05,{ }^{*} p<0.01\right)$. (B) The MFI of CXCR5 on RP105-negative B cells subsets in IgG4-RD with multiple (multiple) and single organ involvement (single) and normal subjects (normal). $\left({ }^{*} \mathrm{p}<0.05\right)$. 
Persistent expression of CXCR5 in plasmablasts (subset 3) may be related to the disappearance of much later subsets 4 and 5 in the peripheral blood and the infiltration of plasmablasts into the multiple target organs in IgG4-RD. The differential expression of CXCR5 in IgG4-RD and SLE may influence the differential distribution pattern of RP105-nagetive B cell subsets in the peripheral blood. The results also suggest that targeting chemokine (CXCR5) and its ligands (CXCL13) is a possible strategy in the therapy for patients with refractory IgG4-RD.

Although our study of differential CXCR5 expression shows statistical significance, it is still a pilot study in small numbers of patients. Studies in the larger population of the patients are required.

Syuichi Koarada, Satoko Tashiro, Yukiko Tokuda, Yukihide Ono, Yuri Sadanaga, Rie Suematsu, Nobuyuki Ono, Akihide Ohta, Yoshifumi Tada

Division of Rheumatology, Faculty of Medicine, Saga University, Saga, Japan

Correspondence to Dr Syuichi Koarada, Division of Rheumatology, Faculty of Medicine, Saga University, 5-1-1, Nabeshima, Saga 849-8501, Japan; koarada@cc. saga-u.ac.jp

\section{Competing interests None.}

Patient consent Obtained.

Ethics approval The Ethics Committees of Saga.
Provenance and peer review Not commissioned; internally peer reviewed.

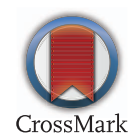

To cite Koarada S, Tashiro S, Tokuda Y, et al. Ann Rheum Dis 2015;74:e32.

Received 23 December 2014

Accepted 28 December 2014

Published Online First 20 January 2015

Ann Rheum Dis 2015;74:e32. doi:10.1136/annrheumdis-2014-207207

\section{REFERENCES}

1 Fox RI, Fox CM. IgG4 levels and plasmablasts as a marker for IgG4-related disease (IgG4-RD). Ann Rheum Dis 2015;74:1-3.

2 Wallace ZS, Mattoo H, Carruthers M, et al. Plasmablasts as a biomarker for IgG4-related disease, independent of serum IgG4 concentrations. Ann Rheum Dis 2015;74:190-5.

3 Koarada S, Tashiro S, Tokuda Y, et al. Subsets of RP105-negative plasmablasts in IgG4-related disease. Ann Rheum Dis 2014;73:e65.

4 Koarada S, Tada Y, Suematsu R, et al. Phenotyping of P105-negative B cell subsets in patients with systemic lupus erythematosus. Clin Dev Immunol 2012;2012:198206.

5 Koarada S, Tashiro S, Nagao N, et al. Increased RP105-Negative B Cells in IgG4-Related Disease. Open Rheumatol J. 2013;23:55-7.

6 Loetscher P, Moser B. Homing chemokines in rheumatoid arthritis. Arthritis Res 2002;4:233-6. 